\title{
Deep reactive ion etching of 'grass-free' widely-spaced periodic 2D arrays, using
} sacrificial structures

\author{
Silvestre, Chantal M.; Nguyen, Vy Thi Hoang; Jansen, Henri; Hansen, Ole
}

Published in:

Microelectronic Engineering

Link to article, DOI:

10.1016/j.mee.2020.111228

Publication date:

2020

Document Version

Peer reviewed version

Link back to DTU Orbit

Citation (APA):

Silvestre, C. M., Nguyen, V. T. H., Jansen, H., \& Hansen, O. (2020). Deep reactive ion etching of 'grass-free' widely-spaced periodic 2D arrays, using sacrificial structures. Microelectronic Engineering, 223, [111228]. https://doi.org/10.1016/j.mee.2020.111228

\section{General rights}

Copyright and moral rights for the publications made accessible in the public portal are retained by the authors and/or other copyright owners and it is a condition of accessing publications that users recognise and abide by the legal requirements associated with these rights.

- Users may download and print one copy of any publication from the public portal for the purpose of private study or research.

- You may not further distribute the material or use it for any profit-making activity or commercial gain

- You may freely distribute the URL identifying the publication in the public portal 


\title{
Deep reactive ion etching of 'grass-free' widely-spaced periodic 2D arrays, using sacrificial structures.
}

\author{
Chantal M. Silvestre ${ }^{*}, 1$, Vy Nguyen ${ }^{1}$, Henri Jansen ${ }^{1}$, and Ole Hansen ${ }^{1}$ \\ ${ }^{1}$ DTU Nanolab, Technical University of Denmark, Kgs Lyngby, DK-2800, Denmark
}

August 18, 2020

\begin{abstract}
We fabricated two-dimensional arrays of periodic, widely-spaced pillars using deep reactive ion etching of silicon. To avoid the formation of micro-grass in the large open areas we used sacrificial structures surrounding the widely-spaced pillars. The use of sacrificial structures results in a denser pattern where the formation of grass is less likely to happen. We were able to remove the sacrificial structures without damaging the main array of pillars by using a modified Bosch process. The roughness remaining after removal of the sacrificial structures was evaluated using optical profilometry. Using this method, we were able to pattern grass-free arrays of widely-spaced $12 \mu \mathrm{m}$ diameter pillars of 9:1 aspect ratio, with hexagonal and square distributions.
\end{abstract}

\section{Introduction}

Anisotropic etching of silicon using deep reactive ion etching (DRIE) is among the key technologies for fabrication of microstructures for a wide range of applications $[1,2,3]$. DRIE processes are often based on the cyclic Bosch process [4]. This process is a two-phase procedure comprising a passivation phase and an etch phase. First, a fluorocarbon (FC) sidewall protection (i.e. passivation phase) is deposited to protect the substrate against etching. Second, the etching phase ideally first removes the protection on horizontal surfaces and then proceeds to etch silicon. The deposition/etching steps are cyclically repeated and this results in a highly anisotropic etching process, alas with significant sidewall scallops. Process control and sidewall morphology can be improved significantly when the Bosch process is modified to a three-phase process: a FC deposition phase, a removal phase that erodes away the FC on horizontal surfaces and a less aggressive etch phase that etches silicon. This three phase procedure, described in [5], is known as DREM (Deposit/Removal/Etch/Multistep).

A critical issue during anisotropic etching of silicon using DRIE is the formation of needle-like structures also know as grass or black silicon. The grass formation is caused by all sorts of micro masking present on the silicon surface. The extrinsic sources of these micro masks are various, such as native oxide or dust on the surface prior to etching [6]. But, micro masking can also occur in the vacuum chamber during the etching process itself. This intrinsic micro masking can either be due to re-deposition of the masking material sputtered by the incoming high energy ions, or from remaining passivation of the surface [7], or from particulates originating from the chamber walls.

The formation of grass is often more pronounced in larger open areas with low aspect ratio than in narrow features with high aspect ratio. This effect is particularly seen when the micro masking originates from the passivation of the surface. Here, the thickness of the passivation layer plays a significant role. In the large spaced areas, where the aspect ratio remains low during the whole etching process, the structures will receive more fluorocarbon species because the cone of incoming fluorocarbon species is wider (Figure 1A (1)). As a consequence, residues will build up thicker than in higher aspect ratio. Thus, during the subsequent removal step, parts of the passivating film may remain on the surface (Figure 1A (2)) causing micro masking when the removal is insufficient on the bottom surface. This results in the formation of particulates during the etching 
(Figure 1A (3)). The particulates will become taller with the increasing number of cycles and will eventually turn into grass (Figure 1A (4)). On the other hand, the deposited fluorocarbon quickly becomes thinner in the narrow spaces as the aspect ratio increases, whereas the removal (which is ideally purely directional), will not decrease as much, thereby ensuring a clean removal of the bottom surface passivation layer after each cycle.
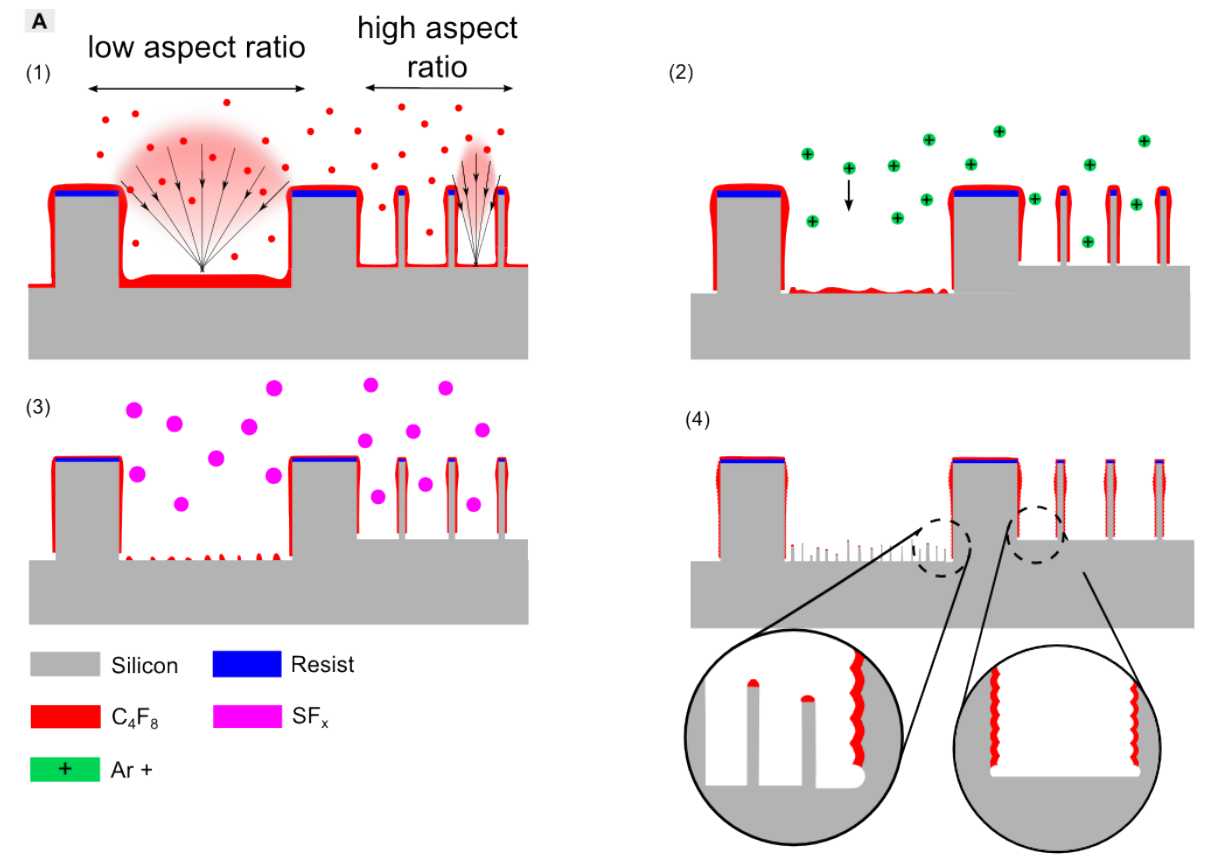

B
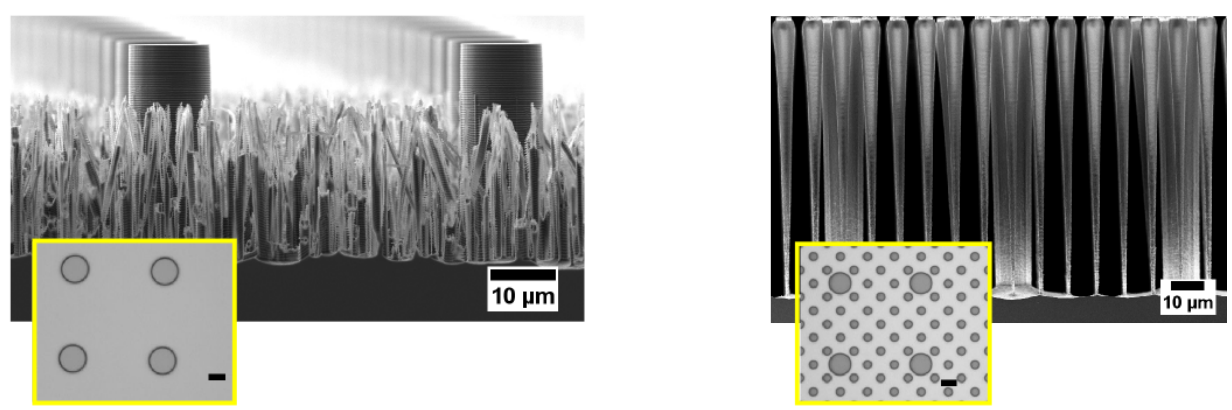

Figure 1: Illustration of the grass formation during DRIE process. A: Deposition step, a thicker FC layer deposits in large open area (2) Removal step, intended for removal of FC on horizontal surfaces. (3) Etching step, the remains of the thicker FC layer in larger areas create micro-masking during the etch step (4) Formation of grass due to micro masking in large open area. B: (left) Electron micrograph showing grass formation in large open areas around $\varnothing 12 \mu m$ pillars. (right) Electron micrograph showing the absence of grass between the same Ø12 $\mu m$ pillars surrounded by $\varnothing 4$ $\mu m$ pillars. Deposition of fluorocarbon from the etching process is visible at the top of the pillars making them look wider at the top. Inserts: micrograph of initial lithography pattern. Scale bar: $10 \mu \mathrm{m}$

It has been repeatedly demonstrated that parameters such as temperature, ICP power loading and pressure can affect the roughness in large open areas $[8],[9],[10]$. When the right balance is struck between the parameters, it is possible to obtain a grass-free bottom surface. However, fine-tuning of the etching parameters is often specific to the reactor in which the recipe was optimised. Therefore, a successful recipe is not necessarily working when applied to another reactor, which may have a different geometry or may show what we call a "memory effect" [11] (i.e. contamination of the chamber due to previous usage). More importantly, even minor changes in the mask layout used might have large effect on grass formation. Therefore, in this paper, we put forward a more generic approach to tackle the formation of grass using sacrificial structures. 


\section{Proposed method}

The model system for this study is an array of widely-spaced pillars, which is used as an electroplating mold for an X-ray Au absorber grating, which is a critical optical element in an X-ray phase contrast imaging setup [12]. We propose to use sacrificial local geometries to achieve grass-free widely-spaced pillar arrays where the spacing is still too large to eliminate the risk of grass formation. By introducing sacrificial structures, we reduce the spacing which prevent grass formation as explained in the introduction.

Local sacrificial geometries have been used by Docker et al. [13] who described the "waffle" technique on a silicon-on-insulator (SOI) wafer. They demonstrated a method for handling large open areas that otherwise would have remained in a low aspect ratio during the dry etching. To free up large areas surrounding their silicon device, the authors added a matrix of sacrificial square holes (waffle) with dimensions that prevented the Aspect Ratio Dependent Etching (ARDE) effect (i.e. the difference in etch rate for features having different aspect ratio) between the sacrificial "waffle" and the device. They used the notching effect ([14]) at the interface of the buried oxide to release the sacrificial structures.

In this study, we investigated a series of sacrificial structures surrounding some widely-spaced pillars, and as to whether the sacrificial structures complied with these three rules:

1. Be dense enough to avoid grass formation.

2. Should not introduce ARDE effect.

3. Be removable while keeping the integrity of the main structures.

The overall difficulty in using sacrificial structures is to find a pattern that has an identical spacing between the structures to avoid etching rate variation across the surface. Each of the sacrificial structures must be placed at an equal distance from each other. Our proposed manufacturing method is illustrated in Figure 2 and requires only a single lithography step, one DRIE step, and one ultrasonic cleaning/removal step. A final oxygen plasma clean for a full removal of the remaining fluorocarbon deposits is optional.

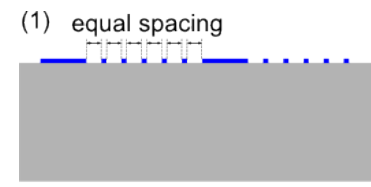

(3)

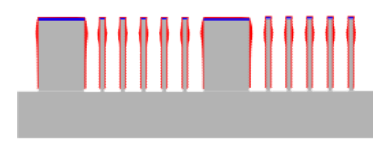

$\mathrm{CF}_{\mathrm{x}} \square$ Resist
(2)

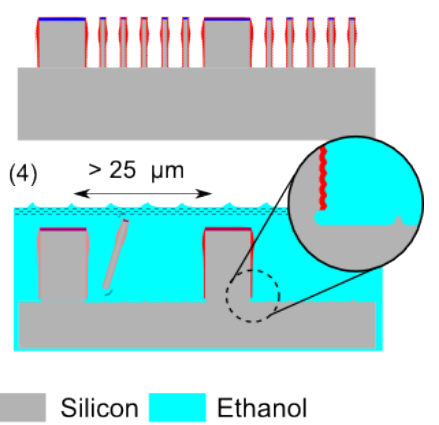

Figure 2: Manufacturing method for reactive ion etching of pillars using an ARDE compensating sacrificial structure design. (1) Photolithography step. (2) Dry etching using optimized DREM process. (3) Isotropic plasma etching to weaken the base of sacrificial structures. (4) Removal of the sacrificial structures in an ultrasonic bath.

The main modification in the etching recipe was an added short oxygen ashing step. A limitation in the DREM process is the accumulation of fluorocarbon on the top part of the structures, which eventually closes the openings and prevent etching. To circumvent this impasse we added an oxygen plasma ashing step to reduce the FC accumulation. This method, named DREAM for Deposit/Removal/Etch/Ashing/Multistep, was proposed by the authors of the DREM process, and is described fully in ref. [15]. Following the cyclic DREAM recipe, we used two additional none-cyclic steps meant to selectively weaken the sacrificial structures. The parameters for the cyclic silicon anisotropic etching process as well as the parameters for the subsequent steps for weakening of the sacrificial structures are listed in Table 1.

Briefly, the method is divided in two distinct phases, (1) a cyclic anisotropic etch phase and, (2) a non-cyclic phase for selective weakening of the sacrificial structures. In the first cyclic etching step, the substrate was 
Table 1: Process parameters for deep reactive ion etching and isotropic etch of the structures

\begin{tabular}{|c|c|c|c|c|c|c|}
\hline & \multicolumn{4}{|c|}{ Anisotropic etching cyclic phase } & \multicolumn{2}{|c|}{ Structure weakening } \\
\hline & Deposition & $\begin{array}{l}\text { Bottom } \\
\text { removal }\end{array}$ & Etch & Ashing & Passivation & $\begin{array}{l}\text { Isotropic } \\
\text { etch }\end{array}$ \\
\hline Time $(\mathrm{s})$ & 1.5 & 0.8 & from 0.6 to 1.1 & 1 & 30 & 95 \\
\hline C4F8 (sccm) & 400 & 5 & 5 & 5 & 400 & 0 \\
\hline SF6 (sccm) & 15 & 15 & 600 & 15 & 15 & 200 \\
\hline $\operatorname{Ar}(\mathrm{sccm})$ & 200 & 250 & 250 & 200 & 200 & 250 \\
\hline $\mathrm{O} 2(\mathrm{sccm})$ & 5 & 5 & 5 & 120 & 5 & 0 \\
\hline Coil power (W) & 3000 & 3000 & 3000 & 3000 & 3000 & 3000 \\
\hline Platen power $(\mathrm{W})$ & 1 & 300 & 1 & 1 & 1 & 1 \\
\hline Pressure (mTorr) & 28 & 8 & 20 & 5 & 25 & 14 \\
\hline Temperature (C) & -19 & -19 & -19 & -19 & -19 & -19 \\
\hline
\end{tabular}

exposed to a $\mathrm{C}_{4} \mathrm{~F}_{8}$ plasma to grow a protective layer of $\mathrm{FC}$. In the next bottom removal step, an argon plasma at 8 mTorr with high platen power was used to clear the FC at the bottom of the pattern. The third cyclic step was a time-ramped $\mathrm{SF}_{6}$ etching step (ramped from $0.6 \mathrm{~s}$ to $1.1 \mathrm{~s}$ ). The last cyclic step was a $1 \mathrm{~s}$ oxygen ashing step to reduce FC accumulation. The cyclic routine was repeated until the desired depth was reached. In the final phase, we passivated the structures for $30 \mathrm{~s}$ in $\mathrm{C}_{4} \mathrm{~F}_{8}$ plasma to ensure good side-wall protection of the pillars to prevent the last isotropic $\mathrm{SF}_{6}$ etch step (meant to weaken the sacrificial pillars) from eroding the silicon pillars.

Finally, the sacrificial structures are removed in an ultrasonic bath of ethanol and rinsed in DI water.

The critical distance at which grass appears depends on the geometry. Figure 3 shows SEM images of periodic structures (Ø $12 \mu \mathrm{m}$ pillars and $3 \mu \mathrm{m}$ lines) with varying spacing $(3,5$, and $7 \mu \mathrm{m})$ between structures; the SEM images were selected from a wider range of structures etched using our procedure. At the smallest spacing $(3 \mu \mathrm{m})$ grass is essentially absent while severe grass formation is seen at $7 \mu \mathrm{m}$ spacing. At $5 \mu \mathrm{m}$ spacing faint traces of grass initiation is seen in the interstitial region between the pillars; thus we recommend that spacing between structures should be at most $3 \mu \mathrm{m}$ for pillars and $6 \mu \mathrm{m}$ for parallel lines. 


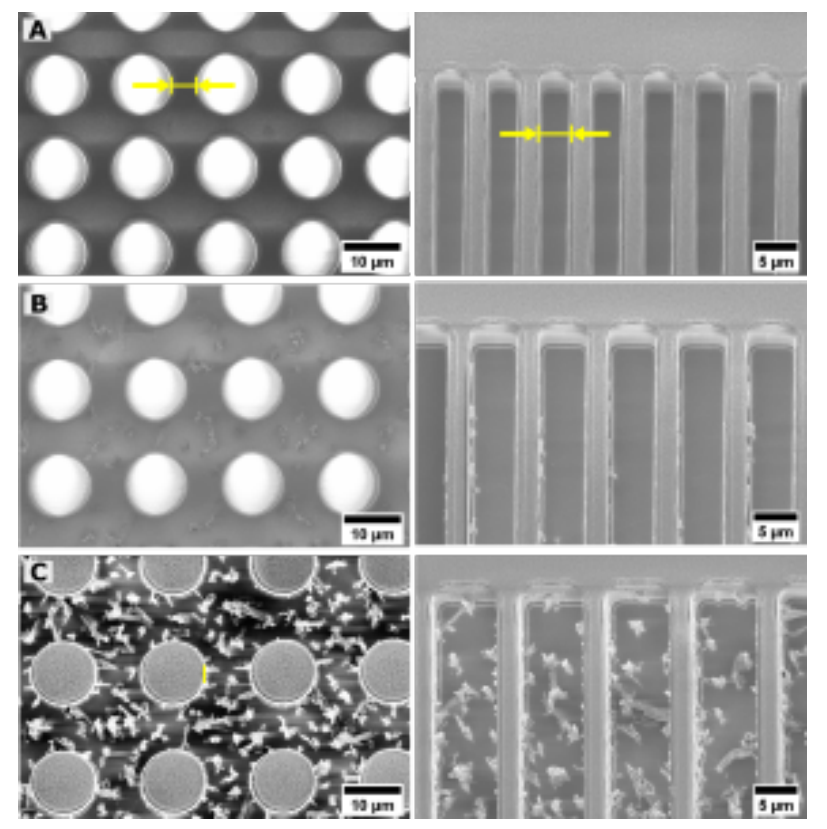

Figure 3: SEM images of etched periodic structures (pillars and lines) with varying distance between the pillars or lines (a) $3 \mu \mathrm{m}$ spacing, (b) $5 \mu \mathrm{m}$ spacing and (c) $7 \mu \mathrm{m}$ spacing. The yellow arrows indicate the smallest distance. Note the grass formation at $7 \mu \mathrm{m}$ spacing.

\section{Materials and methods}

\section{$3.1 \quad$ Sample processing}

The samples were processed on $100 \mathrm{~mm}$ diameter n-type, 1-20 $\Omega \mathrm{cm},(100)$ silicon wafers. The lithography was performed on a $1.5 \mu \mathrm{m}$ thick image reversal resist (AZ5214e) using a maskless i-line aligner (MLA 100 from Heidelberg Instruments Mikrotechnik $\mathrm{GmbH}$ ) and a dose of $40 \mathrm{~mJ} / \mathrm{cm}^{2}$. The resist was used in image reversal mode. After the exposure, the samples were baked at $110^{\circ} \mathrm{C}$ for $120 \mathrm{~s}$ followed by a flood-exposure with a dose of $210 \mathrm{~mJ} / \mathrm{cm}^{2}$. Finally, the samples were developed for $90 \mathrm{~s}$ in a TMAH-based solution (AZ $726 \mathrm{MIF}$ $2.38 \% \mathrm{TMAH}$ in water) to reveal the pattern. The diameter of the pillars were $9 \mu \mathrm{m}$ with $27 \mu \mathrm{m}$ spacing, or $12 \mu \mathrm{m}$ diameter with $50 \mu \mathrm{m}$ spacing. The width of the sacrificial structures varied between $1.5 \mu \mathrm{m}$ and $4 \mu \mathrm{m}$ depending on the design tested. Prior to etching, the samples were manually cleaved into approximately $1 \times$ $1 \mathrm{~cm}^{2}$ chips, and bonded onto the centre of an alumina coated $100 \mathrm{~mm}$ diameter carrier wafer using Galden HT-270 oil. The alumina coating proved to be resistant to the etching plasma, and the carrier wafer could be re-used throughout the experiments. The deep reactive ion etching was done in a SPTS system (DRIE Pegasus), and the resist was used as the masking material. The etching parameters, reported in Table 1, were kept constant throughout the study. After the etching process step and without unloading the sample, we ran a passivation step for $30 \mathrm{~s}$ followed by an isotropic etch step to weaken the base of the sacrificial structures. The chips were then unloaded and characterised.

\subsection{Measurement methods}

After etching, the chips were manually cleaved in half using a diamond pen. The first half of the chip was placed in an ethanol solution and ultrasonicated for $2 \mathrm{~min}$ at low power. The height of the pillars was evaluated from the cross-section using scanning electron microscopy (SEM, Supra V60 from Zeiss) and the bottom height difference was measured using an optical profiler (OP, PLu Neox 3D from Sensofar). To ensure full access for measurement of the bottom surface morphology, the pillars were mechanically removed prior to the OP measurement. The removal was performed using mechanical rubbing of the surface with a cotton bud. The broken structures, which remained on the surface, were cleaned up using ultrasound. Figure 4A shows a schematic cross-section prior to the removal of the sacrificial structures. The cross-section schematic gives an 


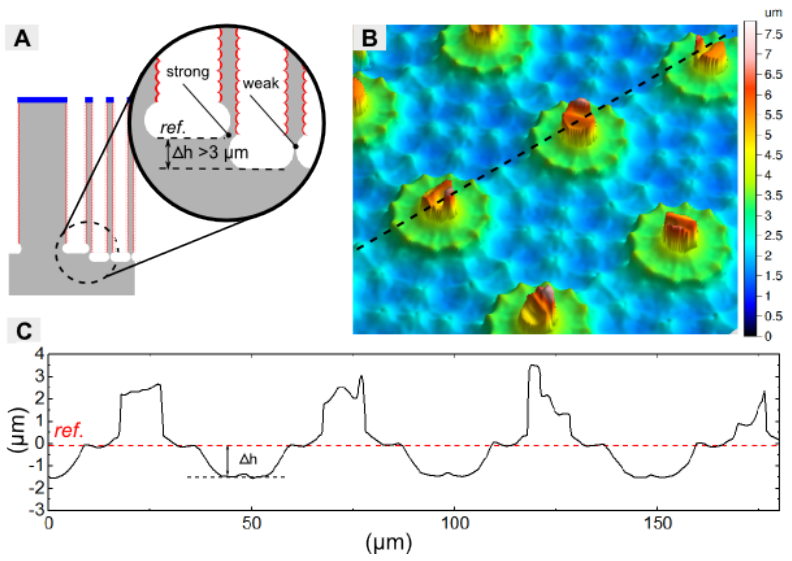

Figure 4: Illustration of the OP characterization. (A) Schematic sample cross-section illustrating a height difference between two adjacent areas. If $\Delta h$ is above $3 \mu \mathrm{m}$, the base of the structure is too stable to be removed with ultrasound. (B) $3 D$ optical profilometry of an array of pillars after removal of the sacrificial structures and mechanical removal of the pillars. (C) Line profile across an array of pillars along the dashed line in $B$. The reference height is set at the base of the main pillars.

example of the height difference between the base of a sacrificial structure and the base of a pillar; the base of the pillar is used as the reference height. We observed that if the height difference $(\Delta \mathrm{h})$ between two adjacent areas exceeded $3 \mu \mathrm{m}$, the sacrificial structures were more difficult to remove using the described method. In this case, to successfully remove the sacrificial structures a longer isotropic etch would be needed to weaken the base. However this could also impair the stability of the main pillars. Therefore, in this experiment, we defined an "acceptance window for height difference" i.e. a bottom height difference in the range between 0 and $3 \mu \mathrm{m}$. An OP measurement in the form of a colour-coded height map of the surface is shown in $4 \mathrm{~B}$, while $4 \mathrm{C}$ shows the surface height as a line scan extracted from the colour map along the dashed line in $4 \mathrm{~B}$. The reported bottom height measurements are performed on an average of six measurements across each scan direction.

\section{Experiments}

In this section, we present the results of 6 series of designs to evaluate the ARDE compensation. The series from 1.x to 3.x have square distribution of circular pillars of $12 \mu \mathrm{m}$ diameter, spaced with a pitch of $50 \mu \mathrm{m}$. The series 4.x has a square distribution of square pillars of $12 \mu \mathrm{m}$ side length, with a pitch of $50 \mu \mathrm{m}$. The series 5.x reports the results of hexagonal pillars of $12 \mu \mathrm{m}$ width placed in a hexagonal distribution, and spaced with a diagonal pitch of $50 \mu \mathrm{m}$. Finally, series $6 . x$ reports the results of circular pillars of $9 \mu \mathrm{m}$ diameter with hexagonal distribution and $27 \mu \mathrm{m}$ pitch.

\subsection{Design \#1.0}

In the initial design, we used $4 \mu \mathrm{m}$ diameter sacrificial pillars arranged in concentric rings around the main $12 \mu \mathrm{m}$ diameter pillars as illustrated in the insert of Figure 5A. We etched three samples with 88, 176 and 220 cycles corresponding to pillar heights of approximately 40,80 and $90 \mu \mathrm{m}$, respectively. The height difference at the bottom surface as function of the etched pillar height from the two line scan directions is shown in Figure 5A. Figure 5A shows that, at least up to $90 \mu \mathrm{m}$ pillar height, the sacrificial structures perform well with regards to grass formation and do not introduce ARDE problems. Both scan directions showed similar bottom height difference, and the standard deviation was negligible. However, occasionally the sacrificial pillars could collapse and stick together or to the main pillars as illustrated in Figure 5B. In the latter case, the removal of the sacrificial pillars using an ultrasonic bath became impossible. Therefore, this design did not fulfil the requirement of the sacrificial structures to be removed while keeping the integrity of the main structures. We suggest that the collapse of the sacrificial pillars occurred during unloading of the samples 
when the chamber is vented. The venting could cause mechanical vibration of the sacrificial pillars, and force them into contact after which they may stick and collapse. If the collapse occurred during etching process, the etch result (ARDE effect, profile, etc.) would have been severely affected, but no such effect was observed.
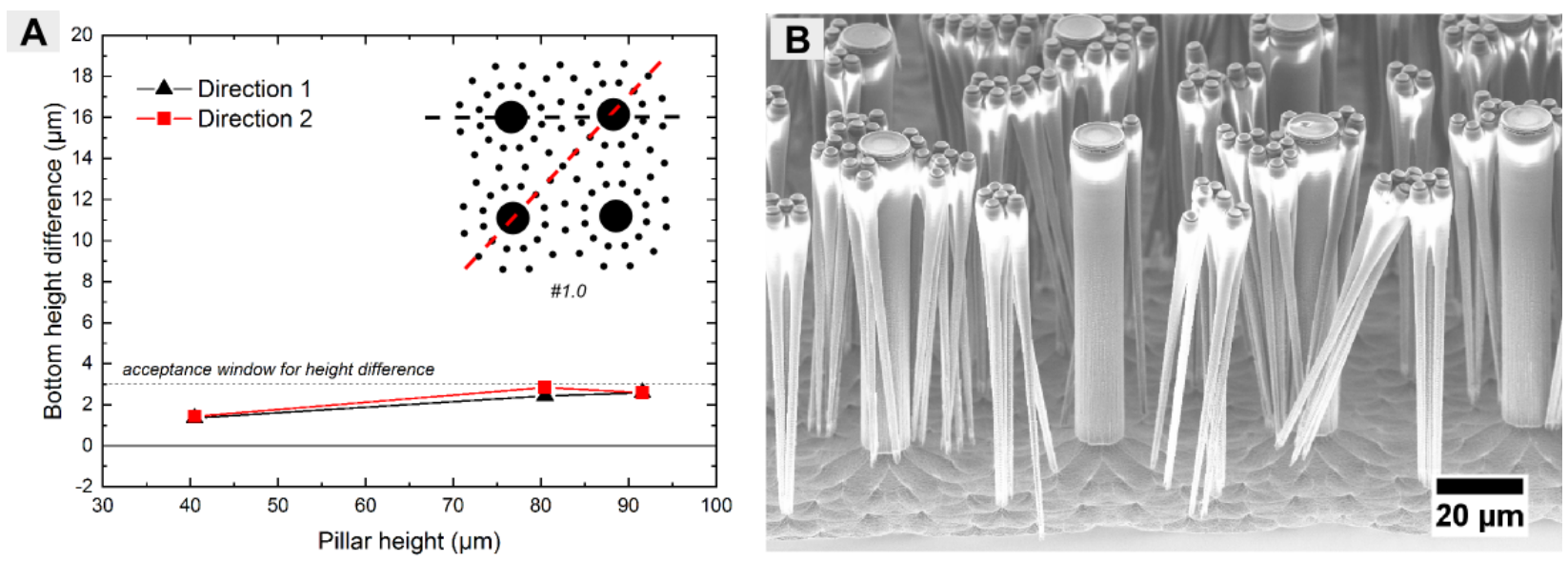

Figure 5: A: Bottom height difference in directions 1 (lateral) and 2 (transverse) of the pillar sacrificial structure. The resist pattern and the scan directions are illustrated in the upper right corner. B: SEM image of sacrificial pillars collapsed after unloading from the DRIE tool. The pillar are covered with fluorocarbon caused by the cyclic dry etching process.

\subsection{Designs \#2.x}

To protect the main pillars against collapse of the sacrificial pillars, we replaced some of the sacrificial pillars of design \#1.0 with sacrificial concentric rings. When using concentric rings, the radial spacing between the edge of the main pillar and the edge of the sacrificial ring is constant, which will prevent ARDE within the rings. The sacrificial rings are mechanically more stable and do not collapse. In the design \#2.x series, we kept some sacrificial pillars in the interstitial area between the rings, and we tested three different distributions of the sacrificial interstitial pillars. The dimensions of the mask pattern are reported in Table 2 and the designs are illustrated in the inserts of Figure 5. The diameter and the pitch of the main pillars remained unchanged at $12 \mu \mathrm{m}$ and $50 \mu \mathrm{m}$, respectively.

Table 2: Design dimensions of the sacrificial structures for designs \#2.x

\begin{tabular}{cccccc}
\hline & \multicolumn{2}{c}{$\begin{array}{l}\text { Interstitial } \\
\text { dot pattern }\end{array}$} & & \multicolumn{2}{c}{$\begin{array}{c}\text { Concentric } \\
\text { ring dimensions }\end{array}$} \\
\cline { 2 - 3 } \cline { 5 - 6 } ID & Qty & $\varnothing$ & & Spacing & Width \\
\hline$\# 2.0$ & 5 & $4 \mu \mathrm{m}$ & & $6.4 \mu \mathrm{m}$ & $2 \mu \mathrm{m}$ \\
$\# 2.1$ & 5 & $4 \mu \mathrm{m}$ & & $6.4 \mu \mathrm{m}$ & $2 \mu \mathrm{m}$ \\
$\# 2.2$ & 9 & $4 \mu \mathrm{m}$ & & $6.4 \mu \mathrm{m}$ & $2 \mu \mathrm{m}$ \\
\hline
\end{tabular}

The measured height differences reported in Figure 6 showed that designs \#2.0 and \#2.1 did not satisfy the ARDE requirement. In these two cases, the height difference was above the $3 \mu \mathrm{m}$ threshold and the standard deviation on the height difference was significant. The sacrificial structures could not be removed. The height difference was also quite dependent on the scan directions. In designs \#2.0 and \#2.1, the larger height difference occurred in the interstitial area (scan direction 2) where the distribution of the sacrificial pillars did not sufficiently prevent ARDE. In designs \#2.0 and \#2.1, the dots did not cover the interstitial area equally, this caused a local gap variation which resulted in strong ARDE as seen in the optical profiler maps illustrated in Figure 7A. The height difference was reasonable within the interstitial area and perfectly balanced within the concentric rings. However, between these two zones, the height difference was significant (larger than $3 \mu$ ) and caused ultrasonic removal problems. 

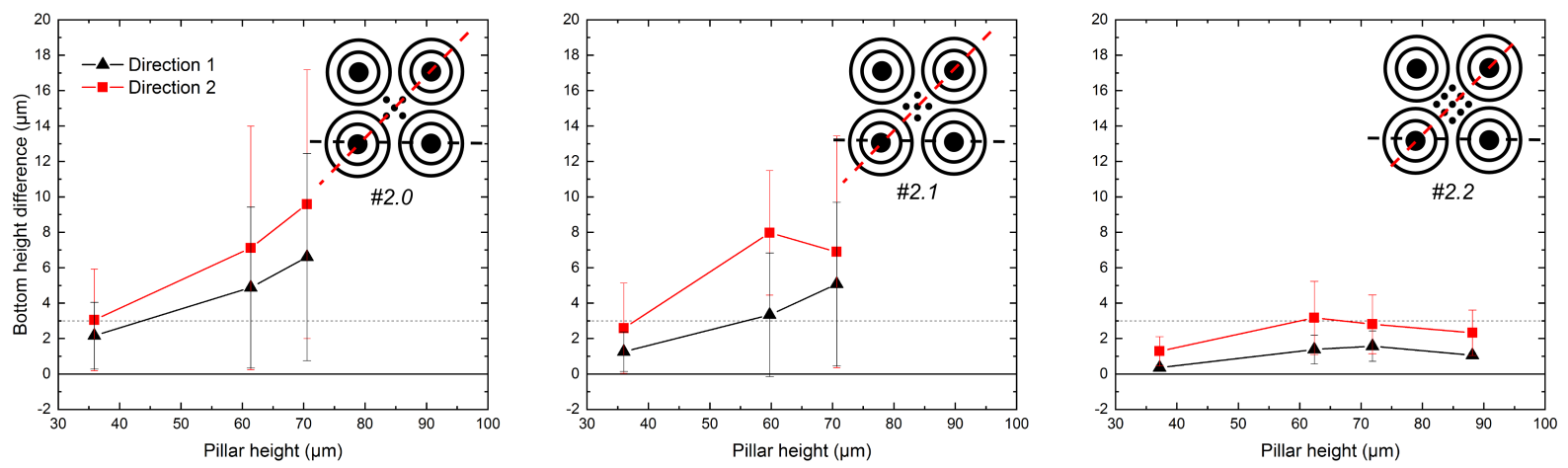

Figure 6: Bottom height measurement for square distribution of circular pillars with combined sacrificial rings and sacrificial dots arranged as illustrated in the inserts for the three designs. All samples were etched for 88, 176, or 220 cycles; for design \#2.2 an additional experiment at 320 cycles was included.

Increasing the number of dots from 5 to 9 , as done in design \#2.2, reduced ARDE, and the bottom height difference remained below our $3 \mu \mathrm{m}$ threshold across the full pattern. Using this design, it was possible to remove the sacrificial structures while keeping the integrity of the array as seen in Figure 7B. This series shows that using sacrificial rings instead of pillars solves the problem of sacrificial pillars sticking to the main structures. The concentric rings compensate nicely for the ARDE inside the rings, however, the interstitial area between the four main pillars remains a zone of difficulty. Design \#2.2, is the only successful in this series and fulfils all the requirements.

\subsection{Designs \#3.x}

In this design series, the interstitial sacrificial pillars were replaced by an interstitial sacrificial cross in another attempt to reduce the variation in height difference within the interstitial area. The dimensions of the mask patterns are listed in Table 3 while inserts in Figure 8 show the layouts. The diameter and the pitch of the main pillars remained unchanged at $12 \mu \mathrm{m}$ and $50 \mu \mathrm{m}$, respectively.

Table 3: Design dimensions of the sacrificial structures for designs \#3.x

\begin{tabular}{ccclcc}
\hline & \multicolumn{2}{c}{$\begin{array}{c}\text { Interstitial } \\
\text { cross pattern }\end{array}$} & & \multicolumn{2}{c}{$\begin{array}{c}\text { Concentric } \\
\text { ring dimensions }\end{array}$} \\
\cline { 2 - 3 } \cline { 5 - 6 } ID & Length & Width & & Spacing & Width \\
\hline$\# 3.0$ & 17 & $2 \mu \mathrm{m}$ & & $6.4 \mu \mathrm{m}$ & $2 \mu \mathrm{m}$ \\
$\# 3.1$ & 27 & $2 \mu \mathrm{m}$ & & $6.4 \mu \mathrm{m}$ & $2 \mu \mathrm{m}$ \\
$\# 3.2$ & 33 & $2 \mu \mathrm{m}$ & & $6.4 \mu \mathrm{m}$ & $2 \mu \mathrm{m}$ \\
\hline
\end{tabular}

The height differences reported in Figure 8 show that design \#3.0 did not satisfy the ARDE requirement. In this design, the length of the cross left large open areas which were etched faster than the surrounding pattern, as seen in OP map in Figure 9A.

When increasing the length of the cross from $17 \mu \mathrm{m}$ to $27 \mu \mathrm{m}$ like in design \#3.1, the height variation in both the scanned directions dropped to a value lower than the $3 \mu \mathrm{m}$ threshold. Despite having an average height difference below the threshold, removal of the sacrificial structures could not be performed in this case. The 3D optical profiler result revealed that the zones at the four extremities of the cross (red arrow in 9A), which were not part of either of the scan direction, were subject to a faster etch. Consequently, the height difference was locally too high and restricted the removal of the outer sacrificial ring. To obtain a better ARDE compensation and perform a clean removal of the sacrificial structures, we extended the length of the cross to $33 \mathrm{\mu m}$ in design \#3.2. An example of the bottom height difference and the clean array is illustrated in Figure 9B.

To evaluate whether design \#3.2 could achieve deeper structures, we etched an additional chip with 450 


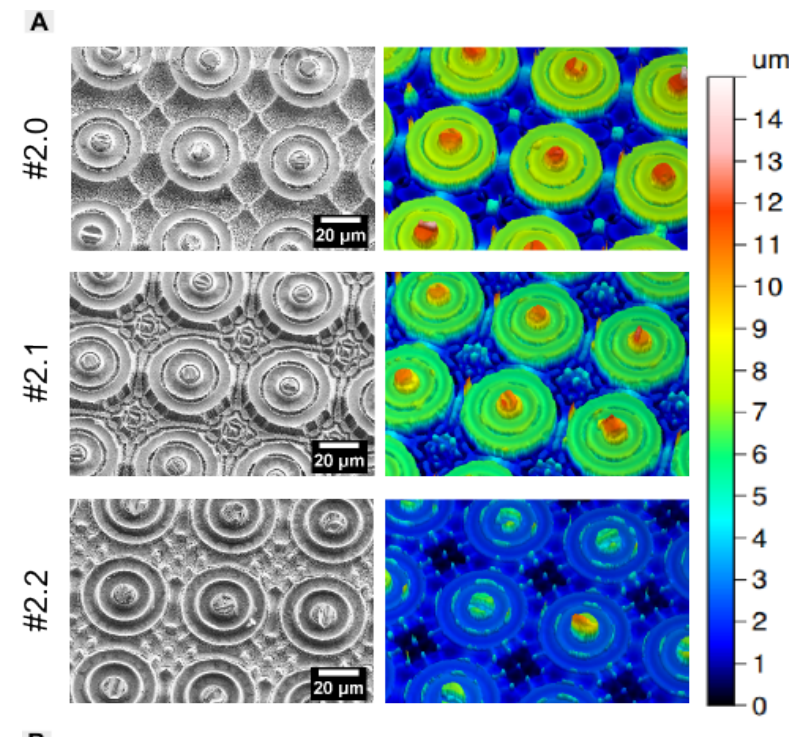

B

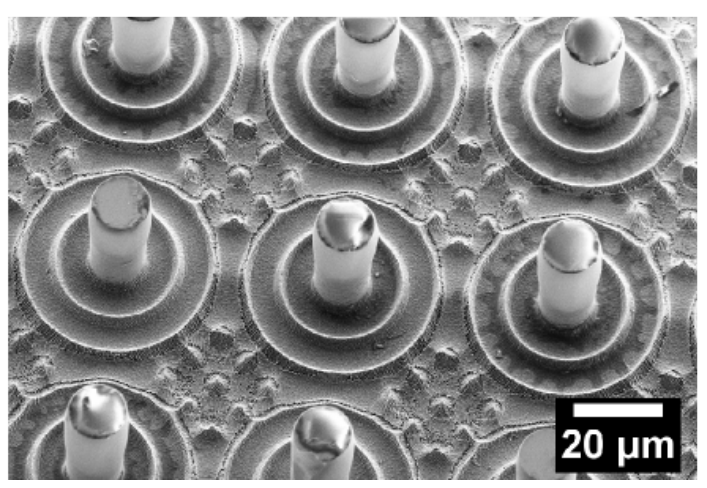

Figure 7: A: optical profiler 3D topography of the bottom surfaces after mechanical removal of the pillars for a etch of 88 cycles, and SEM image of the clean array after ultra-sonication of the sacrificial structures. Scale bar: 20 4 m. B: Widely-spaced arrays of pillars using design \#2.2
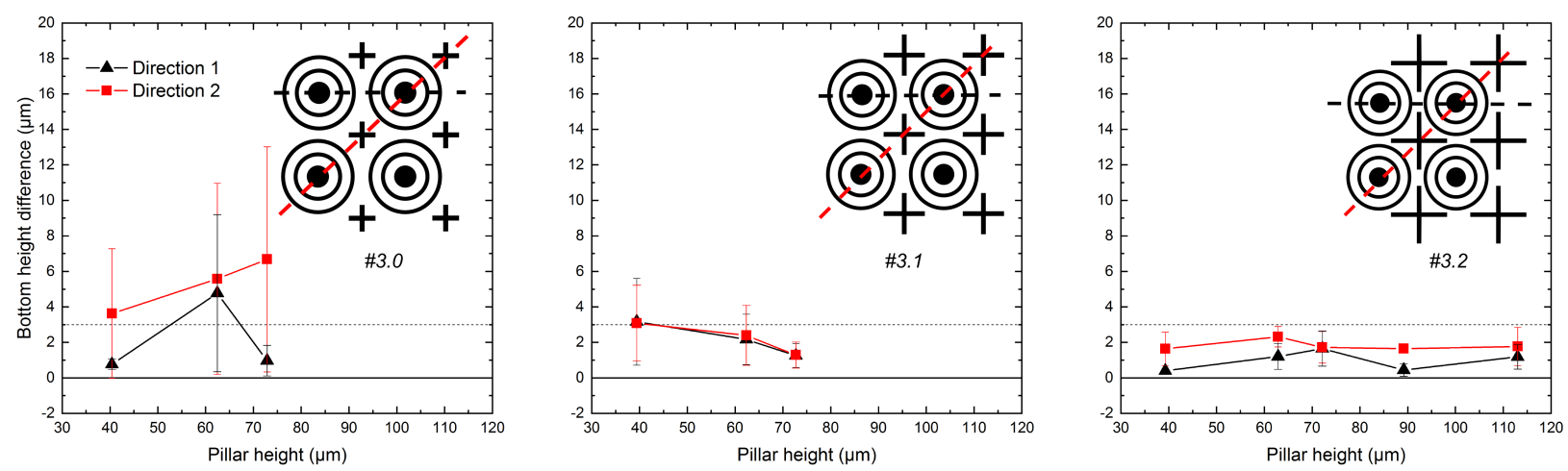

Figure 8: Bottom height measurement for square distribution of circular pillars with sacrificial rings and crosses combined. All samples were etched for 88, 176, or 220 cycles; for design \#3.2 additional experiments at 320 and 450 cycles were done.

cycles. The measured height for this successful sample was $110 \mu \mathrm{m}$, which gave an aspect ratio of 9:1. We observed a slight overetch at the top of the structures due to masking material erosion. The bottom 
height difference returned a value below $3 \mu \mathrm{m}$ in both directions, which was still below our threshold. This experiment suggests that using this design could lead to even higher aspect ratio pillars. Just like design \#2.2, design \#3.2 satisfies all our requirement.
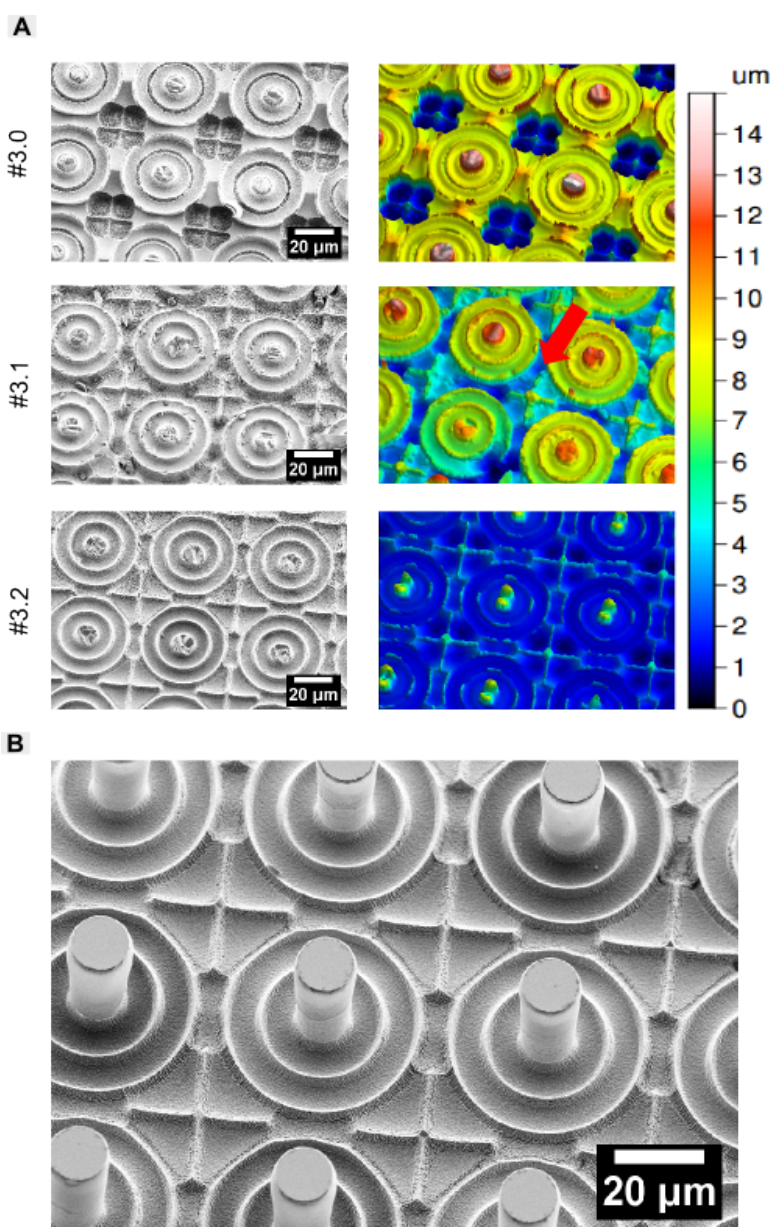

Figure 9: A: optical profiler 3D topography of the bottom surfaces after mechanical removal of the pillars for a etch of 88 cycles, and SEM image of the clean array after ultra-sonication of the sacrificial structures.Scale bar: $20 \mu m$. B: Widely-spaced arrays of pillars using design \#3.2

\subsection{Designs \#4.x}

In series \#4.x, the round pillars were replaced by square pillars of $12 \mu \mathrm{m}$ side length and $50 \mu \mathrm{m}$ pitch. We also replaced the sacrificial circular rings with concentric sacrificial square rings. When using square shapes, the size of the interstitial area becomes smaller than when circular structures are used, since the structures and the array then have the same shape. We expect that by reducing the interstitial area we can reduce the difficulty in finding appropriate sacrificial structures. However, when using concentric square instead of concentric circular pillars, it is no longer possible to keep all the distances identical within the concentric square rings. While the distance between two parallel surface is constant (in our case $5.4 \mu \mathrm{m}$ ), the distance at the 4 corners is increased by a factor of $\sqrt{2}$. This will induce a local change in the etch depth at the corners.

We studied the effect of a sacrificial dot in the interstitial area on the bottom height difference. The dimensions of the designs are shown in Table 4 while the layouts are illustrated in the inserts of Figure 10.

We successfully created widely-spaced arrays of pillars using design \#4.0, up to a pillar height of $68 \mu \mathrm{m}$, or aspect ratio 5.6:1. The removal of the sacrificial structure was successful despite the fact that the average bottom height difference was well above our defined window of height acceptance, at least in one of the scan 
Table 4: Design dimensions of the sacrificial structures for designs \#4.x

\begin{tabular}{ccccc}
\hline & $\begin{array}{c}\text { Interstitial } \\
\text { pattern }\end{array}$ & & \multicolumn{2}{c}{$\begin{array}{c}\text { Concentric } \\
\text { ring dimension }\end{array}$} \\
\cline { 2 - 2 } \cline { 5 - 6 } ID & Dot $\varnothing$ & & $\begin{array}{c}\text { Parallel } \\
\text { spacing }\end{array}$ & Width \\
\hline$\# 4.0$ & $0 \mu \mathrm{m}$ & & $5.4 \mu \mathrm{m}$ & $2 \mu \mathrm{m}$ \\
$\# 4.1$ & $2 \mu \mathrm{m}$ & & $5.4 \mu \mathrm{m}$ & $2 \mu \mathrm{m}$ \\
$\# 4.2$ & $3 \mu \mathrm{m}$ & & $5.4 \mu \mathrm{m}$ & $2 \mu \mathrm{m}$ \\
\hline
\end{tabular}
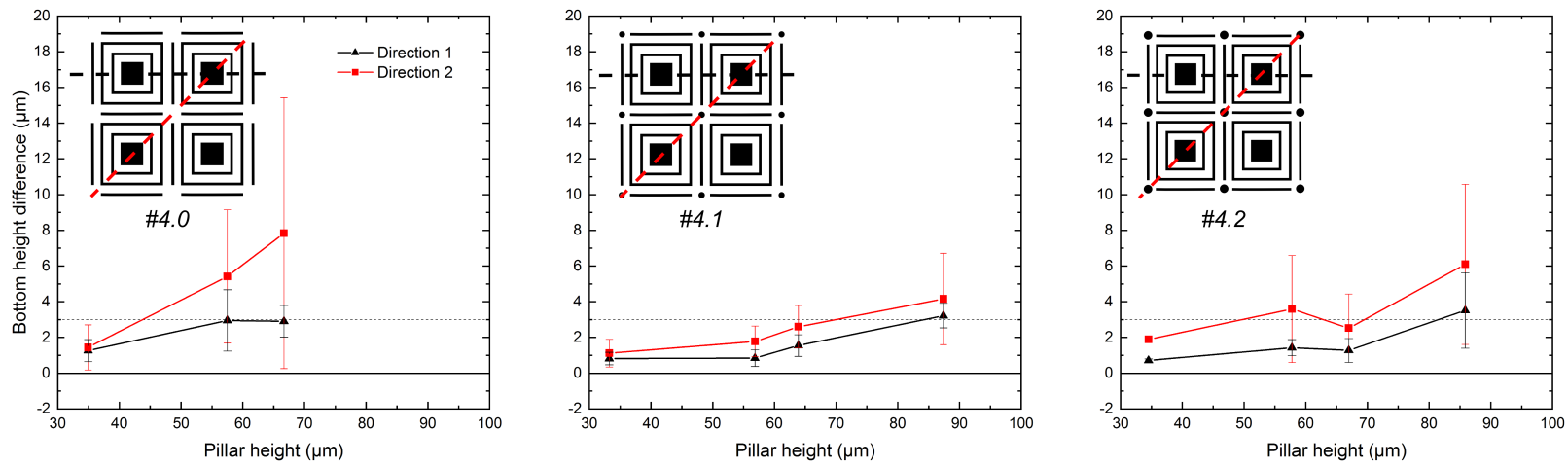

Figure 10: Bottom surface height difference for a square distribution of square pillars combined with sacrificial squarerings. All samples were etched for 88, 176, and 220 cycles; for designs \#4.1 and \#4.2, additional experiments with 320 cycles were performed.

directions. The OP measurement illustrated in Figure 11A shows where the height difference was the largest.

In design \#4.1 we inserted a $2 \mu \mathrm{m}$ diameter dot in the interstitial area to evaluate whether it would reduce the ARDE observed in \#4.0. Here, the first two runs (88 and 176 cycles) showed an average height difference and a variation, below our $3 \mu \mathrm{m}$ limit, see Figure 10. In these runs, the sacrificial structures could be removed successfully to create widely-spaced arrays of pillars. However, the last two runs (220 and 320 cycles) returned an average height close to, or beyond the acceptance window, with a variation exceeding the $3 \mu \mathrm{m}$. In these last two runs, a clean removal of the sacrificial structures could not be performed.

In design \#4.2, a larger $3 \mu \mathrm{m}$ diameter dot was placed in the interstitial area. Figure 10 shows that the bottom surface height difference is below the $3 \mu \mathrm{m}$ threshold at lower pillar height. This low value can also be seen in Figure 11A. The height difference eventually increased above the threshold when the pillar height was increased. The removal of the sacrificial structures was impossible already from a pillar height of $58 \mu \mathrm{m}$.

Overall, we observed that design \# 4.0 was better than \#4.2 even though the bottom height difference was generally too high. Figure 11B illustrated an array of square pillars using this design \#4.0. Although seemingly conflicting with the design rule, which states that $\Delta \mathrm{h}$ should be below $3 \mu \mathrm{m}$ to successfully remove the sacrificial structures, design \#4.0, is the only successful design in this series.

\subsection{Design \#5.x}

The interstitial zone between four pillars distributed in a square pattern still proved to be critical. For circular pillars, we showed that a sacrificial cross in the interstice between four pillars gave satisfying results to a depth of more than $100 \mu \mathrm{m}$, however a slight change in geometry had large consequences on the observed ARDE. Using square-shaped pillars and square sacrificial ring proved to still be challenging even though the interstitial zone was better in avoiding ARDE. We expect that in using a hexagonal distribution of the pillars instead of a square distribution we would eliminate the interstitial area and therefore reduce the ARDE.

We tested two designs with hexagonal pillars distributed in a hexagonal pattern. The width of the hexagonal 
A

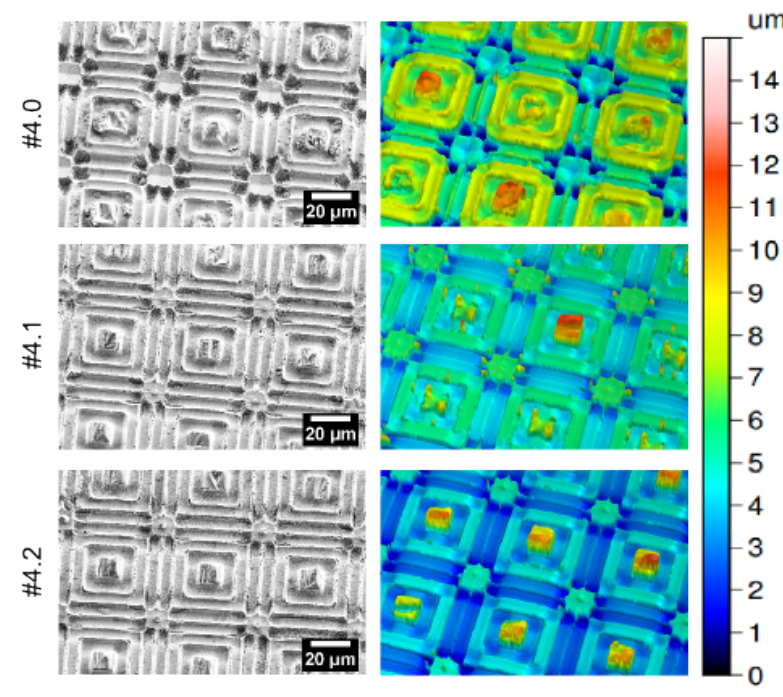

B

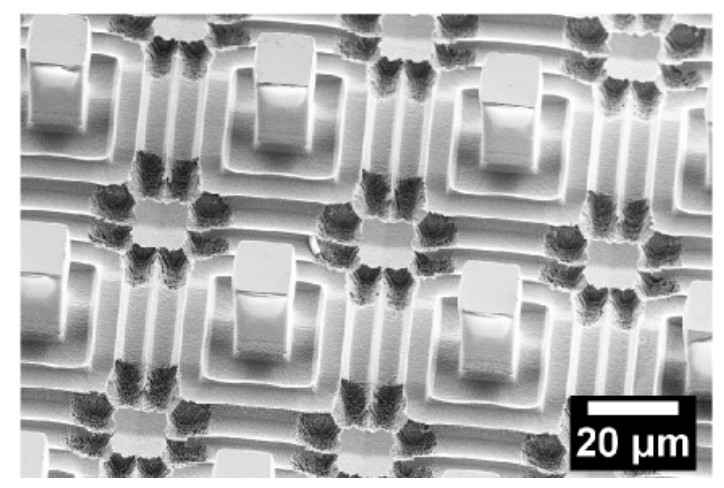

Figure 11: A: optical profiler 3D topography of the bottom surfaces after mechanical removal of the pillars for a etch of 88 cycles, and SEM image of the clean array after ultra-sonication of the sacrificial structures. Scale bar: $20 \mu m$. B: Widely-spaced arrays of pillars using design \#4.0

Table 5: Design dimensions of the sacrificial structures for designs \#5.x

\begin{tabular}{cccccc}
\hline & \multicolumn{2}{c}{ Lines } & & \multicolumn{2}{c}{ Side length } \\
\cline { 2 - 3 } \cline { 5 - 6 } ID & Spacing & Width & & $\begin{array}{c}\text { Inner } \\
\text { hexagon }\end{array}$ & $\begin{array}{c}\text { Outer } \\
\text { hexagon }\end{array}$ \\
\hline$\# 5.0$ & $7.5 \mu \mathrm{m}$ & $1.5 \mu \mathrm{m}$ & & $15 \mu \mathrm{m}$ & $24.5 \mu \mathrm{m}$ \\
$\# 5.1$ & $7.5 \mu \mathrm{m}$ & $1.5 \mu \mathrm{m}$ & & $15 \mu \mathrm{m}$ & $20 \mu \mathrm{m}$ \\
\hline
\end{tabular}

main pillars was $10 \mu \mathrm{m}$, and the horizontal pitch was $50 \mu \mathrm{m}$. The dimensions of the sacrificial structures are reported in Table 5. Similarly to the square rings, the hexagonal rings will show a change in the spacing at their 6 corners which corresponds to an increase of $\frac{2}{3} \sqrt{3} \approx 1.15$. This is closer to the ideal factor 1 . It is therefore expected to observe less ARDE at the 6 corners than in the square geometry.

Design \#5.0 consisted of a hexagonal ring around the main pillars, surrounded by a continuous honeycomb structure. In this case, the interstitial area is absent since all the lines could be placed at equal distances. The result of the bottom height difference in both scan directions illustrated in Figure 12 revealed an average height difference mostly below $1 \mu \mathrm{m}$, with very low variation, which suggests that the design is close to ideal in regard to avoiding ARDE. The 3D optical measurement in Figure 13A also indicate an ideal ARDE. The main downside of this design rests with the removal of the sacrificial continuous honeycomb, which came off as a single sheet, and partially damaged the main pillars as seen in Figure 13B. 

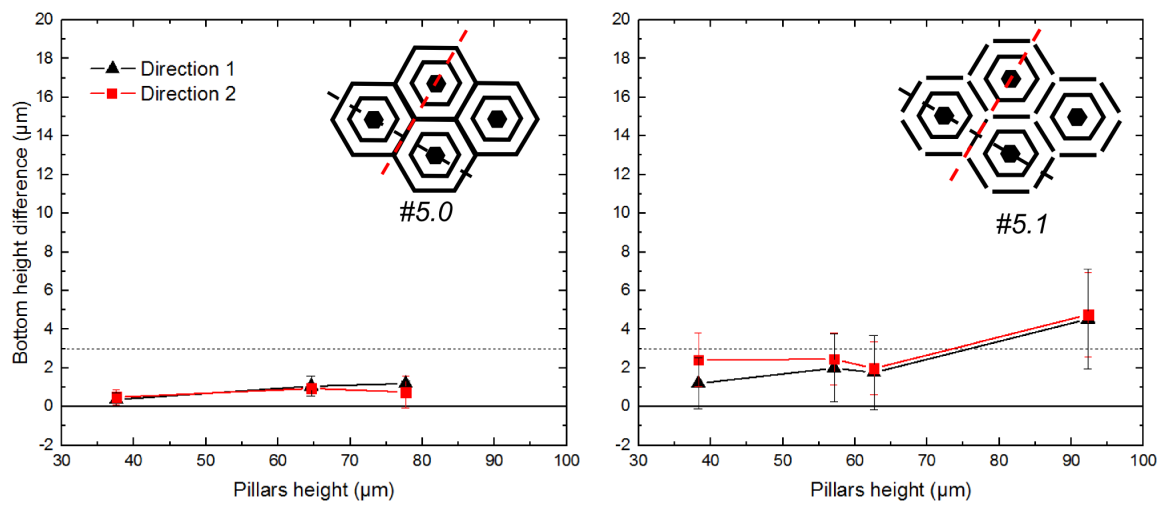

Figure 12: Bottom height measurement for hexagonal distribution of hexagonal pillars with hexagonal sacrificial structures; design \#5.0 has a full sacrificial honeycomb while an interrupted sacrificial honeycomb is used in design \#5.1 as illustrated in the inserts. All samples were etched for 88,176 and 220 cycles; for design \#5.1 an additional experiment with 320 etch cycles was performed.

A
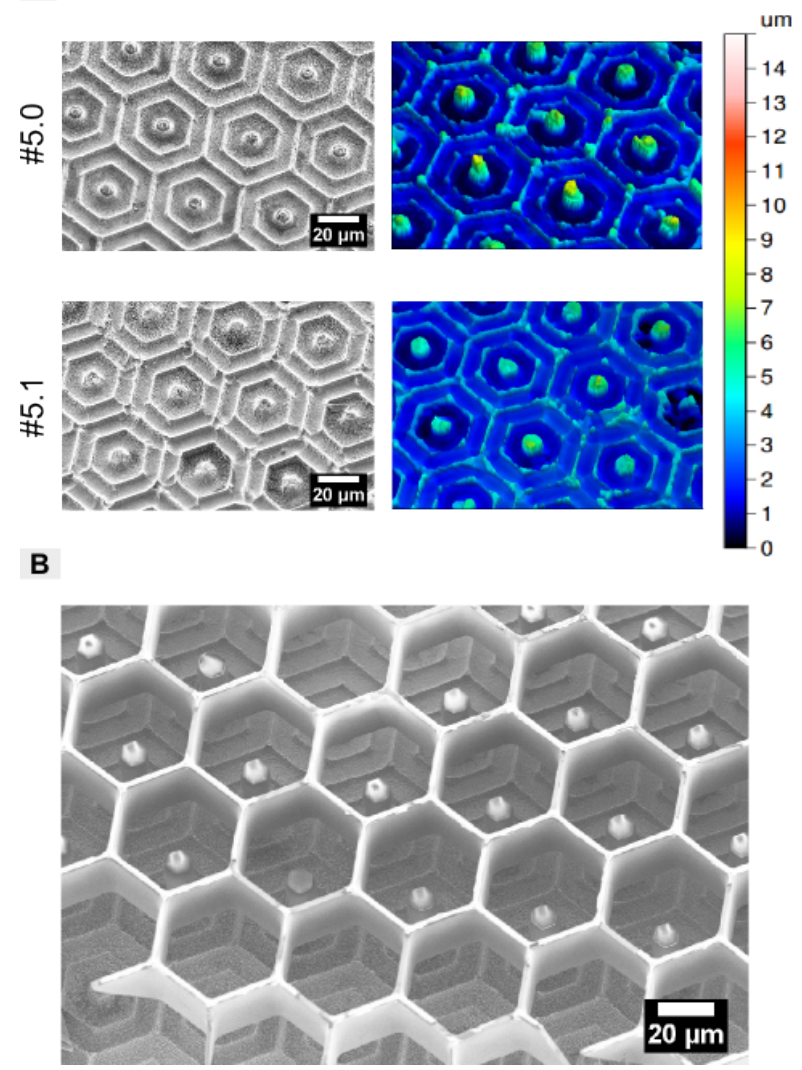

Figure 13: A: optical profiler 3D topography of the bottom surfaces after mechanical removal of the pillars for a etch of 88 cycles, and SEM image of the clean array after ultra-sonication of the sacrificial structures. B: Electron image of the single honeycomb sheet being lifted-off in design \#5.0.

In the attempt to reduce the damages caused by the single honeycomb sheet, we added a gap at the interstitial area to facilitate the removal ability. In design \#5.1, the gap was created by reducing the side length of the outer hexagonal dimensions. The size of the gap was chosen to be of similar dimensions as in the previous designs tested. The results reported in Figure 12 indicate no significant ARDE, up to a pillar height of $63 \mu \mathrm{m}$ 
(AR 6.3:1) and sacrificial structures could be removed. However, when etched for 320 cycles, the bottom height difference suddenly increased considerably, which indicates that the ARDE is no longer balanced.

\subsection{Designs \#6.x}

As seen with designs series \#5.x, the hexagonal distribution offers the least ARDE variation across the pattern due to the significant reduction of the difficult interstitial area. In series \#6.x we decided to combine the hexagonal distribution with circular pillars. To do so, we distributed circular pillars in an hexagonal pattern. The pillars were placed closer with a pitch of $27 \mu \mathrm{m}$. We used a sacrificial ring around the main pillars with a distance of $5.4 \mu \mathrm{m}$ from the edge of the pillars. Unlike the square and the hexagonal shape, the distance from the pillar to the edge of the circular ring is not reduced by any geometrical factor. The distance remains constant, which totally avoids ARDE within the ring. The dimensions of the mask patterns are reported in Table 6 .

The two layouts differed only in the unavoidable interstitial area caused by the circular ring. In design \#6.1 we introduced a $2 \mu \mathrm{m}$ sacrificial dot to reduce ARDE between the interstitial area and the area inside the sacrificial ring.

Table 6: Design dimensions of the sacrificial structures for designs \#6.x

\begin{tabular}{ccrcc}
\hline & \multirow{2}{*}{$\begin{array}{c}\text { Interstitial } \\
\text { pattern }\end{array}$} & & \multicolumn{2}{c}{$\begin{array}{c}\text { Concentric circle } \\
\text { dimensions }\end{array}$} \\
\cline { 2 - 2 } \cline { 5 - 5 } ID & Dot $\varnothing$ & & Spacing & Width \\
\hline$\# 6.0$ & $0 \mu \mathrm{m}$ & & $5.4 \mu \mathrm{m}$ & 1.5 \\
$\# 6.1$ & $2 \mu \mathrm{m}$ & & $5.4 \mu \mathrm{m}$ & 1.5 \\
\hline
\end{tabular}

Figure 14 shows the bottom height difference for the two designs tested. It is clear that, even with hexagonal distribution, the interstitial area remains an issue with regard to ARDE, as seen in Figure 15A design \#6.0. A distinct improvement was obtained when a sacrificial dot was inserted as seen in design \#6.1. In this latter case, the surface height difference was well below the threshold.
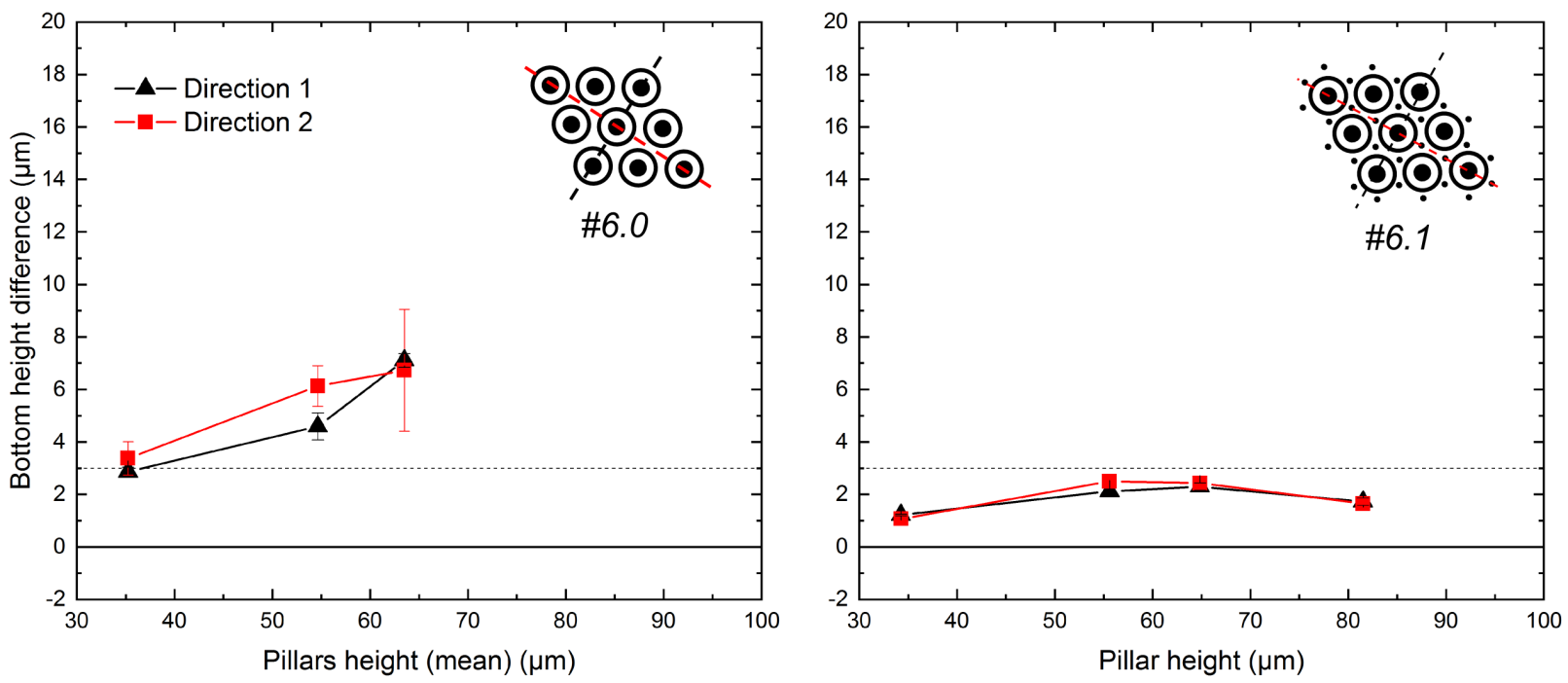

Figure 14: Bottom surface height measurement for hexagonal distribution of circular pillars with sacrificial rings; design \#6.1 also has sacrificial dots, which are missing on design \#6.0 as seen in the inserts. All samples were etched for 88, 176 and 220 cycles; for design \#6.1 an additional experiment with 320 etch cycles was done. 
A

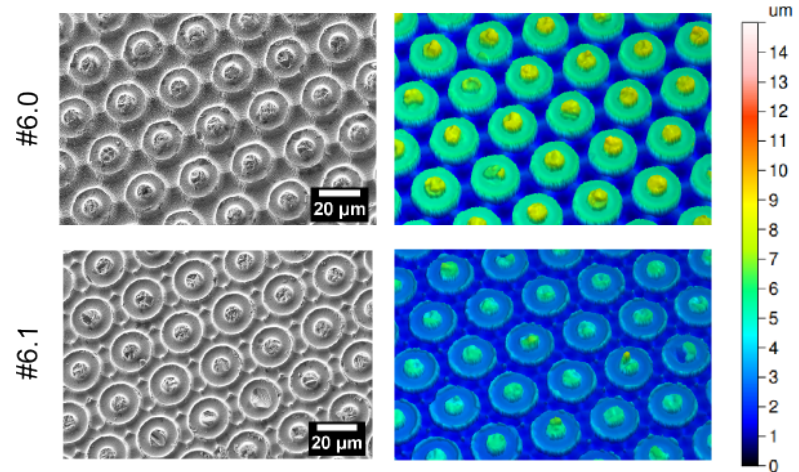

B

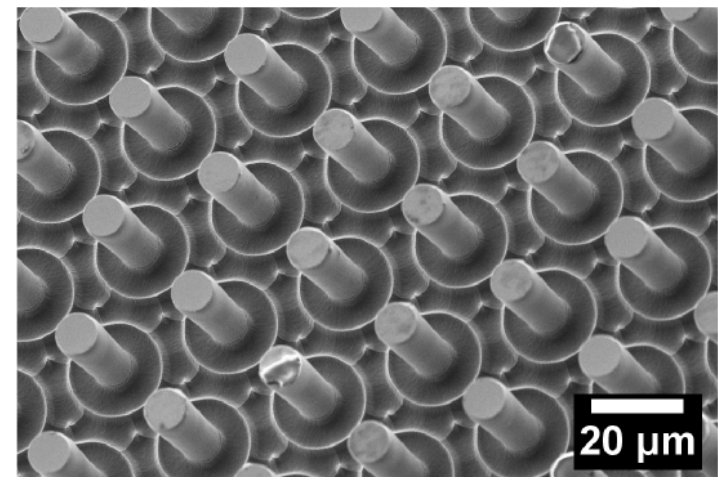

Figure 15: A: optical profiler 3D topography of the bottom surface after mechanical removal of the pillars after 88 cycles of etch, and SEM image of the clean array after ultra-sonication of the sacrificial structures. Scale bar: $20 \mu m$. B: Widely-spaced arrays of pillars using design \#6.1

Design \#6.1 satisfies our condition to create grass-free widely-spaced pillars with a bottom surface roughness below $3 \mu \mathrm{m}$. The sacrificial structures were removed easily while keeping the integrity of the main pillars as seen in Figure 15B. The maximum height tested and obtained with design \#6.1 was $82 \mu \mathrm{m}$, which led to pillars with 9:1 aspect ratio. Figure 15A shows the optical profilometer maps of the bottom surfaces after mechanical removal of the pillars of designs \#6.0 and \#6.1.

\section{Discussion}

The study demonstrates that sacrificial structures can effectively suppress grass formation during DRIE of structures (here widely spaced pillars) that would suffer from severe grass formation if sacrificial structures are omitted. Indeed, all the sacrificial structure designs that were tested resulted in grass-free surfaces. The successful application of this grass-suppression strategy, however, depends on several factors: the stability of the sacrificial structures, the symmetry of the patterns, and good control of ARDE.

Poor stability of the sacrificial structures may prevent removal of the sacrificial structures by post-etching, since they may stick to the main structures, the pillars, as seen with Design \#1.0. Too stable sacrificial structures, on the other hand, may prevent damage-free removal of the sacrificial structures as seen with the continuous honeycomb sacrificial structure of Design \#5.0.

Sacrificial structures with low enough stability to allow damage-free removal will inevitably leave more or less open interstitial areas (depending on symmetry) that give rise to issues with ARDE control, unless proper sacrificial structures are also added there. These issues are illustrated in Designs \#2.x to \#6.x, and are thus present both when pillars and arrays have similar symmetry and when they have different symmetry.

Symmetry is important as illustrated in the completely hexagonal Design \#5.0, which is ideal in all aspects 
except for the too high stability of the sacrificial structure. Here ARDE is very well controlled simply due to the complete absence of interstitial areas.

In fact, based on our findings a simple rule can be set for an ideal symmetry. Every feature should have an identical feature placed at an equal spacing from it. In Figure 16 we give some examples of good and bad symmetries, and in Figure 17 we propose good candidates for further optimisation of the designs and a generic approach. In a generic approach, the intended structures should always be surrounded by guarding ring that matches the shape as correctly as possible, the rest of the space should be filled in with pillars that prevent ARDE.

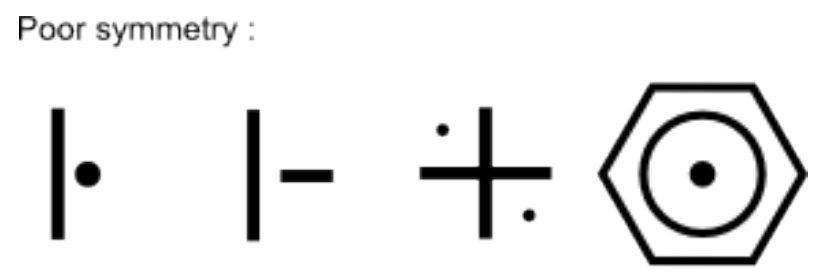

Good symmetry :

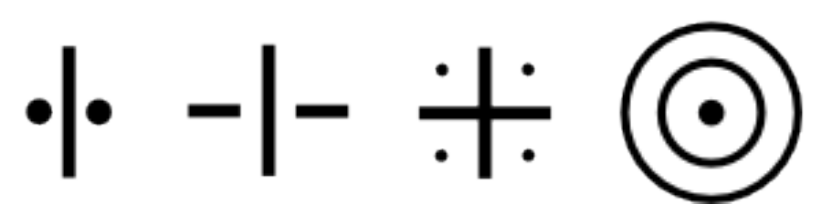

Figure 16: Example of geometry exhibiting poor and good symmetry for ARDE compensation

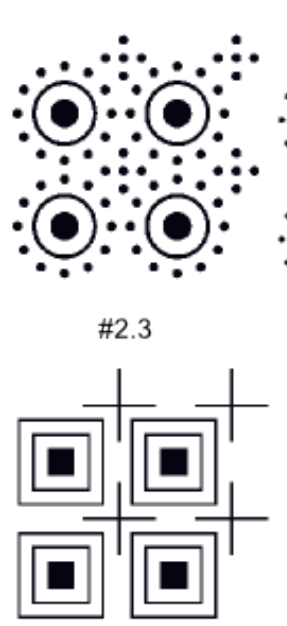

\#4.3

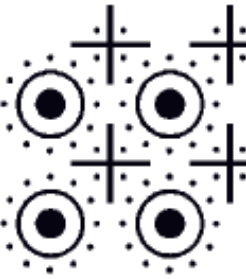

\#3.3

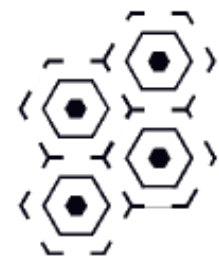

$\# 5.3$

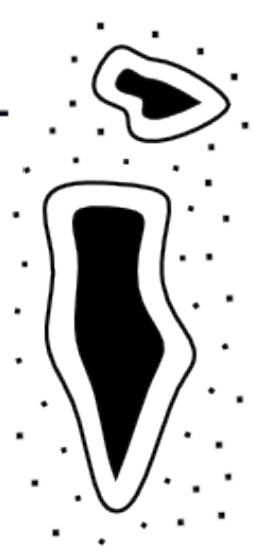

generic design

Figure 17: Proposed designs based on ideal symmetry

\section{Conclusion}

The use of sacrificial structures has shown to be beneficial in assisting the DRIE of large areas where the risk of grass formation is increased. In our experiment, we have an array of widely-spaced pillars which encounters this grass formation issue when performing DRIE. By using sacrificial structures, we reduced the spacing surrounding the pillars, and could suppress the formation of grass without the need of fine-tuning the parameters of the recipe.

We have investigated the effect of sacrificial structures as a method to reduce ARDE and avoid formation of 
grass. All experiments were done using the same DRIE recipe while the geometry of the sacrificial structures was varied. The results are that for pillars distributed in a square pattern, the ARDE significantly impacts the etch rate in the interstitial area between four pillars. Various sacrificial structures were tested in this interstitial area to evaluate and reduce the impact of ARDE. We also showed that the interstitial area problem was reduced significantly with a hexagonal distribution of pillars where perfect symmetry can be obtained. Using the method proposed in this work, we successfully fabricated 9:1 aspect ratio circular pillars, distributed in square and hexagonal pattern.

\section{Acknowledgement}

The authors acknowledge the Innovation Fund Denmark for financial support.

\section{References}

[1] Bingdong Chang, Chen Zhou, Abebe Tilahun Tarekegne, Yuanqing Yang, Ding Zhao, Flemming Jensen, Jörg Hübner, and Henri Jansen. Large Area Three-Dimensional Photonic Crystal Membranes: SingleRun Fabrication and Applications with Embedded Planar Defects. Advanced Optical Materials, 7(2):1-9, 2019.

[2] Frederik Stöhr, Henning Friis Poulsen, Hugh Simons, Jörg Hübner, Jonas Michael-Lindhard, Ole Hansen, Flemming Jensen, and Anders Clemen Jakobsen. Sacrificial structures for deep reactive ion etching of high-aspect ratio kinoform silicon x-ray lenses. Journal of Vacuum Science 83 Technology B, Nanotechnology and Microelectronics: Materials, Processing, Measurement, and Phenomena, 33(6):062001, 2015.

[3] Konstantins Jefimovs, Lucia Romano, Joan Vila-Comamala, Matias Kagias, Zhentian Wang, Li Wang, Christian Dais, Harun Solak, and Marco Stampanoni. High-aspect ratio silicon structures by displacement Talbot lithography and Bosch etching. Proceedings of SPIE, March 2017:101460L, 2017.

[4] Robert Bosch Gbmh. Franz Laermer, Andrea Schilp. Method of anisotropically etching silicon, US5501893A Patent, 1993.

[5] Bingdong Chang, Pele Leussink, Flemming Jensen, Jörg Hübner, and Henri Jansen. DREM: Infinite etch selectivity and optimized scallop size distribution with conventional photoresists in an adapted multiplexed Bosch DRIE process. Microelectronic Engineering, 191:77-83, 2018.

[6] Henri Jansen, Han Gardeniers, Meint De Boer, Miko Elwenspoek, and Jan Fluitman. A survey on the reactive ion etching of silicon in microtechnology. Journal of Micromechanics and Microengineering, 6(1):14-28, 1996.

[7] Henri Jansen, Meint De Boer, Johannes Burger, Rob Legtenberg, and Miko Elwenspoek. BSM 2 The effect of mask material and loading on the RIE of deep silicon trenches. Journal of Micromechanics and Microengineering, 27(1095):475-480, 1995.

[8] KyuBong Jung, WooJin Song, Hyun Woo Lim, and Caroline Sunyong Lee. Parameter study for silicon grass formation in Bosch process. Journal of Vacuum Science ES Technology B, 28(1):143-148, 2010.

[9] Henri V. Jansen, Meint J. de Boer, and Michael Curt Elwenspoek. The black silicon method vi: high aspect ratio trench etching for mems applications. In Proceedings IEEE Ninth Annual International Workshop on Micro Electro Mechanical Systems 1996, MEMS '96, pages 250-257. IEEE Computer Society, 21996.

[10] M. Mehran, Z. Sanaee, M. Abdolahad, and S. Mohajerzadeh. Controllable silicon nano-grass formation using a hydrogenation assisted deep reactive ion etching. Materials Science in Semiconductor Processing, 14(3-4):199-206, 2011.

[11] Hayden K. Taylor, Hongwei Sun, Tyrone F. Hill, Ali Farahanchi, and Duane S. Boning. Characterizing and Predicting Spatial Nonuniformity in the Deep Reactive Ion Etching of Silicon. Journal of The Electrochemical Society, 153(8):C575, 2006. 
[12] E.S. Dreier, C. Silvestre, J. Kehres, D. Turecek, M. Khalil, J.H. Hemmingsen, O. Hansen, J. Jakubek, R. Feidenhans'l, and U.L. Olsen. Virtual subpixel approach for single-mask phase-contrast imaging using Timepix3. Journal of Instrumentation, 14(01):C01011-C01011, 2019.

[13] P. T. Docker, P. K. Kinneil, and M. C.L. Ward. Development of the one-step DRIE dry process for unconstrained fabrication of released MEMS devices. Journal of Micromechanics and Microengineering, 14(7):941-944, 2004.

[14] Toshihisa Nozawa, Takashi Kinoshita, Tetsuya Nishizuka, Akira Narai, Takayoshi Inoue, and Akimitsu Nakaue. The electron charging effects of plasma on notch profile defects. Japanese Journal of Applied Physics, 34(4):2107-2113, 1995.

[15] Bingdong Chang. Technology Development of 3D Silicon Plasma Etching Processes for Novel Devices and Applications. Ph.D. thesis, Technical University of Denmark, 2018. 